\title{
Analyzing experimental data using the Rasch model
}

\author{
TOM VERGUTS, PAUL DE BOECK, and GERT STORMS \\ University of Leuven, Leuven, Belgium
}

\begin{abstract}
We present a method for studying experimental data based on a psychometric model, the "Rasch model" (Rasch, 1966; Thissen \& Steinberg, 1986). We illustrate the method with the use of a data set in the field of concept research. More specifically, we investigate whether a conjunctive concept can be seen as an additive combination of its constituents. High correlations between model and data are obtained, but a formal goodness-of-fit test indicates that the model does not completely account for the data. We then alter the Rasch model in such a way as to capture our idea of why the model deviates from the data. This results in higher correlations and a strong increase in goodness-of-fit. It is concluded that our ideas, as incorporated in the model, adequately summarize the data. More generally, this research illustrates that applying the Rasch model and altering it according to one's hypotheses is an excellent way to analyze experimental data.
\end{abstract}

In the perception literature, considerable attention has been paid to the fuzzy logic model of perception (FLMP; see, e.g., Massaro \& Hary, 1986; Oden, 1977). Recently, Crowther, Batchelder, and $\mathrm{Hu}$ (1995) have established the formal equivalence between this model and a particular psychometric model-namely, the Rasch model (Rasch, 1966; Thissen \& Steinberg, 1986). In this paper, we explore the consequences of this linkage and apply the Rasch model (or, equivalently, the FLMP) to data on conjunctive concepts (see, e.g., Hampton, 1987, 1988; Storms, De Boeck, Van Mechelen, \& Geeraerts, 1993; Storms, De Boeck, Van Mechelen, \& Ruts, 1996). We first present the FLMP model, followed by the (equivalent) Rasch model. We then apply the model to empirical data and discuss its analysis. In the example, both models lead to the same conclusions, although some extra information is provided in the Rasch formulation.

\section{THE FUZZY LOGIC MODEL OF PERCEPTION}

Suppose we perform a letter recognition experiment (e.g., Oden, 1979) and ask participants whether a stimulus $o_{i}$ should be classified as a $G$ or as a $Q$. Furthermore, suppose that $c_{j i}$ indicates the degree to which aspect $j$ is present in stimulus $o_{i}$. If aspect $j$ is present in the letter $G$, the evidence for $G$ with respect to aspect $j$ is equal to $c_{j i}$; otherwise, it is equal to $1-c_{j i}$. The same reasoning applies for the letter $Q$. For example, given that both $G$ and $Q$ are made up of an oval and a line, the first aspect might be "oval is open on the right." The evidence in $o_{i}$ for $G$ with respect to this first aspect is $c_{1 i}$; for $Q$, it is $1-c_{1 i}$. Similarly, the second aspect might be "line is horizontal."

The authors thank Iven Van Mechelen, Norman Verhelst, and Johan Wagemans for their useful comments. Correspondence should be addressed to T. Verguts, Department of Psychology, Tiensestraat 102, B3000 Leuven, Belgium (e-mail: tom.verguts@psy.kuleuven.ac.be)
The evidence for $G$ and $Q$ in stimulus $o_{i}$ is then $c_{2 i}$ and $1-$ $c_{2 i}$, respectively.

To continue with our example: The FLMP predicts that the probability of stimulus $o_{i}$ to be classified as $G$ [formally, $\operatorname{Pr}\left(o_{i}=G\right)$ ] can be written as

$$
\operatorname{Pr}\left(o_{i}=G\right)=\frac{c_{1 i} c_{2 i}}{c_{1 i} c_{2 i}+\left(1-c_{1 i}\right)\left(1-c_{2 i}\right)},
$$

which can be paraphrased by saying that the evidence for $G$ is weighted against the evidence for $Q$. It is clear that if in this model $c_{1 i}$ or $c_{2 i} \rightarrow 1$, then $\operatorname{Pr}\left(o_{i}=G\right) \rightarrow 1$. It can be noticed that Formula 1 is equivalent to the BradleyTerry-Luce model (see, e.g., Luce, 1959). Although the FLMP is stated here as a stochastic model, this is often not the case (Oden, 1977, 1979). We present it in probabilistic terms, however, in order to facilitate comparison with the Rasch model. Parameter estimates for $c_{1 i}$ and $c_{2 i}$ are obtained by the least squares method. We have presented the FLMP in a specific situation - namely, a twofactor, two-alternative experimental design. The paper will be restricted to this case.

\section{THE RASCH MODEL}

In order to show that the Rasch model and the FLMP are equivalent, we rewrite the parameters $c_{1 i}$ as $1 /\left[1+\exp \left(-\theta_{i}\right)\right]$ and $c_{2 i}$ as $1 /\left[1+\exp \left(-\beta_{i}\right)\right]$. This is simply a one-to-one transformation, which implies that the models have essentially the same mathematical structure. The model can now be written as

$$
\operatorname{Pr}\left(o_{i}=G\right) \frac{\exp \left(\theta_{i}+\beta_{i}\right)}{1+\exp \left(\theta_{i}+\beta_{i}\right)},
$$

which equals the Rasch model. It can be seen that, in this model, the higher the parameter $\theta_{i}$ or $\beta_{i}$ is, the higher the probability (Formula 2) will be, and the probability becomes 1 if $\theta_{i}$ or $\beta_{i}$ is at infinity. On the other hand, if $\theta_{i}$ or $\beta_{i}$ tends off to minus infinity, the probability (Formula 2) 
tends off to 0 . Furthermore, the model is completely compensatory in the sense that for an arbitrary value $\theta_{i}\left(\beta_{i}\right)$, by making $\beta_{i}\left(\theta_{i}\right)$ large or small enough, one can always get the probability (Formula 2) arbitrarily close to 1 or 0 , respectively.

One sees that the re-parametrizations $\theta \rightarrow \theta+D$ and $\beta \rightarrow \beta-D$ (with $D$ an arbitrary constant) result in exactly the same formula (2), illustrating the statement that different solutions of the parameters are only an additive constant away from one another. It can be proved that this is the only way to arrive at new solutions (Andersen, 1980). In other words, fixing a value of $D$ makes the model identified.

There are three differences between the Rasch model as stated in Formula 2 and its usual formulation. First, in the Rasch model the argument of the exponent is most often written as $\theta-\beta$, reflecting a competition, not between two identifications of a stimulus, but (in the context of test theory) between an ability parameter $\theta$ for a subject and a difficulty parameter $\beta$ associated with an item. In Formula 2, on the other hand, both factors $\theta$ and $\beta$ contribute positively to a positive response. This difference is, however, only a matter of parametrization.

Second, in Formula 2 no person parameters are involved. Instead of an item parameter (usually denoted by $\beta$ ) and a person parameter (usually denoted by $\theta$ ), this model has two item parameters, one for each $c$ variable involved. The fact that with an increasing number of subjects the number of parameters does not increase allows us to perform the (relatively easy) joint maximum likelihood (JML) method in order to get consistent parameter estimates. The fact that parameters are estimated consistently implies that the estimations can get arbitrarily close to the true values if the number of observations is increased (Andersen, 1980). The consistency follows from the condition as formulated by Haberman (1977) for consistent JML estimation.

A third difference is that cell counts instead of binary $(0 / 1$, right/wrong) responses are modeled. In this way, the model has some affinity with the less well-known Rasch Multiplicative Poisson model (Ogasawara, 1996).

The advantage of the reformulation of the FLMP as a Rasch model is threefold. All three points will be illustrated in the research to be described. First, it allows a more flexible way to alter the mathematical model according to one's substantive interests. Second, and related to the first point, the FLMP is not identified (and, hence, neither is the Rasch model). However, in the FLMP, new solutions are arrived at by the formulas (Crowther et al., 1995, p. 398):

$$
c_{1 i} \rightarrow \frac{c_{1 i}}{1+D\left(1-c_{1 i}\right)}
$$

and

$$
c_{2 i} \rightarrow \frac{c_{2 i}(1+\mathrm{D})}{1+D c_{2 i}}
$$

$D$ again being an arbitrary constant. In the Rasch model, on the other hand, the different solutions are only an additive constant away from one another, making immediately clear which conclusions concerning the data are warranted and which are not. Third, the Rasch formulation allows the construction of formal goodness-of-fit statistics to check the model. (A goodness-of-fit statistic can also be calculated in the FLMP. In practice, however, this is never done, and more is known about Rasch goodness-of-fit statistics than about the corresponding FLMP tests.)

\section{CONJUNCTIVE CONCEPTS}

Recently, a number of authors have been studying conjunctive concepts (e.g., Hampton, 1988; Storms et al., 1993; Storms et al., 1996). A conjunctive concept as it was studied by those authors is simply a concept of the form $X$ s that are also $Y s$, like "sports that are also games" or "birds that are also pets." We will in the following concentrate on "sports that are also games" as an example.

A typical effect in conjunctive concepts is overextension (Hampton, 1988). This means that a low prototypicality for one of the constituent concepts can be compensated by a high value for the other concept. For example, "hide and seek" may be a good example of games but not at all of sports and may nevertheless be considered a reasonable example of games that are also sports.

Another effect that is often found in the literature is the "dominance effect" (Hampton, 1987). This means that one of the two concepts is more important in the conjunction; the correlation between the (rated) prototypicality of the dominant concept and the conjunction is larger than the correlation between the prototypicality of the nondominant concept and the conjunction. In sports and games, for example, sports is the dominant concept (Storms et al., 1993).

\section{FLMP/RASCH AND CONJUNCTIVE CONCEPTS}

Suppose that the probability of a concept $o_{i}$ to be classified as belonging to the conjunction can be decomposed into two variables: the degree to which $o_{i}$ belongs to the (fuzzy) ${ }^{1}$ set of sports, and the degree to which $o_{i}$ belongs to the (fuzzy) set of games. Let us suppose that $o_{i}$ belongs to levels $j$ and $k$ of the first and second variable, respectively. Furthermore, let us suppose that a sports parameter $\theta_{j}$ and a games parameter $\beta_{k}$ together determine the degree to which $o_{i}$ is a sport that is also a game. Assume further that

$$
\operatorname{Pr}\left(o_{i} \in \text { conjunction }\right)=\frac{\exp \left(\theta_{j}+\beta_{k}\right)}{1+\exp \left(\theta_{j}+\beta_{k}\right)},
$$

in which the term on the left-hand side denotes the probability that $o_{i}$ will be classified as belonging to the conjunction. This model is again the FLMP/Rasch model. This application of the FLMP/Rasch model differs from 
the letter experiment described earlier in that there are no longer two concepts (like $G$ and $Q$ ) to choose from; the two response possibilities refer to the probability that a stimulus belongs to the conjunctive concept or not. This implies that, while $\theta_{j}$ in Formula 2 indicates the evidence for $G$ with respect to the first variable, the parameter $\theta_{j}$ now represents the evidence for the conjunction, as far as the sports dimension is concerned. Furthermore, while $\beta_{k}$ in Formula 2 indicates the evidence for $G$ with respect to the second variable, the parameter $\beta_{k}$ now represents the games dimension.

It can be seen that the model (Formula 3 ) is compensatory, as discussed before. A very low value $\theta_{j}$, for example, can be compensated by a very high value $\beta_{k}$; the effect is that $\operatorname{Pr}\left(o_{i} \in\right.$ conjunction $)$ can still be high. If we would find a satisfactory fit of the Rasch model, this would be in line with the overextension phenomenon. A bad fit, on the other hand, could indicate that the compensation does not occur for the concepts used, and that, in fact, the conjunctive concept may be truly a logical conjunction of both concepts.

The aforementioned "dominance" effect can also be evaluated if the Rasch model holds. Suppose that $\theta$ is the contribution of the extent to which the item is a sport, and $\beta$, of the extent to which an item is a game. The impact of being a sport is larger than that of being a game if the variance of $\theta$ is greater than that of $\beta$ (Storms et al., 1996). This can be stated independently of the particular scale used, since adding a constant $(D)$ to a scale does not influence the variance, as can be seen in the variance formula $\operatorname{Var}(\theta)=E[\theta-E(\theta)]^{2}$. On the other hand, since the FLMP transformation is not linear, to compare variances on the FLMP scale would not be meaningful. This suggests a way out of a problem stated in Crowther et al.'s (1995) paper. These authors claimed that, because of the transformational freedom involved in the FLMP, comparing the scales (like $c_{1 i}$ and $c_{2 i}$ ) is not meaningful. Comparison is meaningful, however, after parametrization as a Rasch model and comparison of the variances of the scales. In the usual Rasch model formulation [in which the argument of $\exp ($.$) is \theta-\beta$ ] one can also compare the means of the scales, since the nonidentification then amounts to a translation of $\theta$ and $\beta$ in the same direction (i.e., adding a constant $D$ to both $\theta$ and $\beta$ ).

\section{EXPERIMENT}

\section{Stimulus Construction}

In order to obtain estimates of $\theta_{j}$ and $\beta_{k}$, it is most convenient to construct a $J \times K$ matrix where rows $(j=$ $1, \ldots, J)$ indicate the levels of the factor $\theta$ (the values $\theta_{j}$, $J$ levels) and columns indicate the levels of the factor $\beta$ (the values $\beta_{k}, K$ levels). In cell $(j, k)$ we have a stimulus at level $j$ of sports and level $k$ of games. For the $J$ levels of sports and the $K$ levels of games a parameter is used, so in general there are $J+K$ parameters. A given stimulus $i$ has a $\theta_{j}$ and a $\beta_{k}$ parameter, the former correspond- ing to its row and the latter to its column. A list of 66 items was constructed, and 12 participants were asked to judge whether they considered each item to be a sport $(+)$, not a sport $(-)$, or a boundary case $(0)$. Afterward, we asked the same questions about games. Let the number of $+\mathrm{s}$ about sports assigned to item $i$ be denoted as $N_{+i \mathrm{~S}}$, the number of $+\mathrm{s}$ for games for item $i$ as $N_{+i \mathrm{G}}$, and $N_{-i \mathrm{~S}}, N_{-i \mathrm{G}}$ analogously. Each item was then assigned the two scores $t_{i \mathrm{~S}}=N_{+i \mathrm{~S}}-N_{-i \mathrm{~S}}$ and $t_{i \mathrm{G}}=N_{+i \mathrm{G}}-N_{-i \mathrm{G}}$. Using these data, items with the same values $t_{i \mathrm{~S}}$ were considered equal with respect to their sports' prototypicality, and items with the same values $t_{i \mathrm{G}}$ were considered as matched on the games dimension. In this way the stimulus matrix in Table 1 could be constructed, in which + , 0 , and - in the row heading mean a high-, moderate-, and low-value $t_{i \mathrm{~S}}$, respectively. The meaning of the column headings is analogous.

The values $t_{i \text { S }}$ chosen for the rows were (from + to - ) 12,1 , and -12 , respectively, while the $t_{i \mathrm{G}}$ values chosen for the column headings were (from + to - ) $12,-5$, and -12 . Some variation in the $t_{i \mathrm{G}}$ and $t_{i \mathrm{~S}}$ values was tolerated in order to fill up all cells of the matrix completely. The deviation between matched values in a row or column was never more than one. As an example, all items in the first row have the same value $t_{i \mathrm{~S}}=12$, while the items in the first column all have a value $t_{i \mathrm{G}}$ of 11 or 12 .

For the interpretation of the matrix, take for example the cell at row 2 and column 1, the card game "bridge." This item was considered to be only moderately a sport $\left(t_{i \mathrm{~S}}=1\right)$, but definitely a game $\left(t_{i \mathrm{G}}=12\right)$.

We then asked 132 (other) participants to judge whether each of the nine items belonged to the conjunction or not. The data are given in Table 2.

\section{Estimation and Testing of the Model}

A JML estimation program ${ }^{2}$ was applied to these data. Because of the nonidentification described earlier, one parameter had to be restricted, making the number of free parameters equal to five. We constructed the following goodness-of-fit statistic:

\begin{tabular}{clll} 
& \multicolumn{4}{c}{ Table 1 } \\
& \multicolumn{3}{c}{ Stimulus Matrix } \\
\cline { 2 - 4 } Sport & \multicolumn{3}{c}{ Game } \\
\hline+ & badminton & karate & marathon \\
0 & bridge & fishing & walking \\
& hide and seek & invest* & cooking \\
\hline
\end{tabular}

*"Invest" here means "to invest in the stock market."

Table 2

Frequencies of Positive Responses for the Stimuli in Table 1

\begin{tabular}{cccc}
\hline & \multicolumn{4}{c}{ Game } \\
\cline { 2 - 4 } Sport & + & 0 & - \\
\hline+ & 114 & 54 & 39 \\
0 & 58 & 63 & 56 \\
- & 49 & 28 & 25 \\
\hline
\end{tabular}




$$
X^{2}=\sum_{i=1}^{9} \frac{\left(n_{i}-N p_{i}\right)^{2}}{N p_{i}\left(1-p_{i}\right)},
$$

in which $i$ goes over all cells, $n_{i}$ indicates the number of subjects responding "yes" (belongs to the conjunction) to the stimulus in cell $i, N$ indicates the number of subjects $(N=132)$ and $p_{i}$ is the probability (Formula 3$)$ with estimated parameters inserted for the true parameters. One sees that Formula 4 is asymptotically $\chi^{2}$ distributed by the following argument. Each term in Formula 4 is a squared standardized binomial variable; therefore, each term is asymptotically $\chi^{2}$ distributed. Hence, the sum Formula 4 is, by the $\chi^{2}$ addition theorem, also $\chi^{2}$ distributed. There are five parameters to be estimated, and nine data points, so Formula 4 is asymptotically $\chi^{2}$ distributed with $9-5=4$ degrees of freedom. All distributions to be discussed later in this paper are derived in a similar manner.

\section{Results}

The correlation between estimated and observed frequencies of a "yes" response in the nine cells was .84 , which is rather high. The expected frequencies are given in Table 3.

The $X^{2}$ statistic, however, resulted in a value of 54.13, which is highly significant with four degrees of freedom $(p<.001)$. How is such a bad fit to be explained? Inspection of the data suggests that the concepts used are not really compensatory: It seems that both values $t_{i \mathrm{~S}}$ and $t_{i \mathrm{G}}$ must be high for a concept to belong to the conjunction-that is, as high as for badminton in the cell corresponding to row 1 and column 1 . We capture this idea in the model as follows:

$$
\operatorname{Pr}\left(o_{i}=G\right)=\frac{\exp \left(\theta_{i}+\beta_{j}+\delta_{i 1} \delta_{i 2} \omega\right)}{1+\exp \left(\theta_{i}+\beta_{j}+\delta_{i 1} \delta_{i 2} \omega\right)},
$$

in which, by definition, $\delta_{i 1}\left(\delta_{j 1}\right)$ equals 1 if $i(j)=1$ and 0 otherwise. In this model, the parameter $\omega$ is only "active" for the cell at row 1 , column 1 . In this way, the model becomes a mix of a compensatory component $\left(\theta_{i}+\beta_{j}\right)$ and a noncompensatory one $\left(\delta_{i 1} \delta_{i 1} \omega\right)$. Fit of this model would indicate that compensation does occur, but that in order for a really high proportion of "yes" a high value along both dimensions is required. We modified the program in such a way as to estimate the parameters $\theta, \beta$, and $\omega$ of the model (Formula 5). The predictions of this model are given in Table 4. The correlation between observed and predicted values is in this case .98 , while the $X^{2}$ statistic resulted in a value of $9.05(d f=3, p>.01)$.

Table 3

Expected Frequencies Matrix for the Model in Formula 3

\begin{tabular}{cccc} 
& \multicolumn{4}{c}{ Game } \\
\cline { 2 - 4 } Sport & + & 0 & - \\
\hline+ & 89.8 & 63.3 & 53.6 \\
0 & 80.0 & 53.0 & 43.8 \\
- & 52.0 & 29.2 & 22.9 \\
\hline
\end{tabular}

Table 4

Expected Frequencies Matrix for the Model in Formula 5

\begin{tabular}{crcc}
\hline & \multicolumn{4}{c}{ Game } \\
\cline { 2 - 4 } Sport & + & 0 & - \\
\hline+ & 114.0 & 50.9 & 42.1 \\
0 & 66.9 & 59.8 & 50.4 \\
- & 40.1 & 34.4 & 27.5 \\
\hline
\end{tabular}

Table 5

Expected Frequencies Matrix for the Model in Formula 6

\begin{tabular}{crcc}
\hline & \multicolumn{3}{c}{ Game } \\
\cline { 2 - 4 } Sport & + & 0 & - \\
\hline+ & 114.0 & 74.0 & 64.1 \\
0 & 83.9 & 60.2 & 50.5 \\
- & 67.9 & 44.6 & 36.1 \\
\hline
\end{tabular}

Both measures indicate that the parameter $\omega$ greatly contributes to a good fit of the model, and hence that the data are to an important degree noncompensatory.

Since this model fits, it makes sense to ask whether one concept is dominant over the other. We therefore calculate the variance of row and column scale values, $\operatorname{var}(\theta)$ and $\operatorname{var}(\beta)$. These result in 0.190 and 0.065 , respectively. The difference cannot be explained by a difference in variance between the corresponding "physical" scales $t_{i \mathrm{~S}}$ and $t_{i \mathrm{G}}$, since the latter difference goes in the opposite direction $\left[\operatorname{var}\left(t_{i \mathrm{~S}}\right)<\operatorname{var}\left(t_{i \mathrm{G}}\right)\right]$. Hence, sports can be considered to be the dominant concept, in accordance with previous findings (Storms et al., 1993).

We can now go one step further and assume that the psychological scales $\theta$ and $\beta$ are a mere linear transformation of the $t_{i \mathrm{~S}}$ and $t_{i \mathrm{G}}$ scales, respectively. This transformation results in a reduction of the number of parameters and hence a more parsimonious model. The new model is written as

$$
\operatorname{Pr}\left(o_{i}=G\right)=\frac{\exp \left(t_{i \mathrm{~S}} \theta+t_{i \mathrm{G}} \beta+\delta_{i 1} \delta_{i 2} \omega\right)}{1+\exp \left(t_{i \mathrm{~S}} \theta+t_{i \mathrm{G}} \beta+\delta_{i 1} \delta_{i 2} \omega\right)},
$$

in which the only parameters left to be estimated are $\theta$, $\beta$, and $\omega$. We report the expected frequencies for the model (Formula 6) in Table 5.

The correlation between observations and predictions of the model now equals 89 , but the $X^{2}$ statistic resulted in a value of $79.33(d f=6, p<.001)$, which is highly significant, and thus the model (Formula 6) cannot account for the data. Clearly the transformations from the "physical" scales $t_{i \mathrm{~S}}$ and $t_{i \mathrm{G}}$ to the psychological scales $\theta_{i}$ and $\beta_{i}$ are not linear.

In all our models, the argument of $\exp ($.$) is written as$ a linear combination of some parameters. Different linear combinations amount to different hypotheses about the data (compare the models in Formulas 3, 5, and 6). Such a procedure is well known in the Rasch model (as in, for example, the LLTM, see Fischer, 1973, 1995). Since the FLMP does not combine parameters in a linear way, analogous transformations for the FLMP are not straightforward. For example, even a simple operation as 
adding a constant to $\theta$ and $\beta$ amounts to a quite complicated transformation in the FLMP (see Crowther et al., 1995, Formulas 3 and 4).

\section{RMSD Analysis}

A standard way to evaluate model fit in FLMP modeling is to evaluate the root mean squared deviation (RMSD) between observations and predictions (e.g., Oden, 1977); its formula in our case is given by

$$
\mathrm{RMSD}=\sqrt{\frac{\sum_{i}\left(p_{i}-r_{i}\right)^{2}}{9}},
$$

where $r_{i}$ denotes the proportion of subjects who responded positively in cell $i$ (stimulus $i$ ). We applied Formula 7 to our data for the models in Formulas 3, 5, and 6, resulting in values of $.102, .041$, and .134 , respectively. Although it is not very clear how one should evaluate RMSD values, informal comparisons with RMSDs published in the literature (Massaro \& Hary, 1986; Oden, 1977, 1979) show that the first and the third are high, and the second, moderately low. These results, then, point in the same direction as does the formal analysis discussed above.

Computing both magnitude-of-discrepancy measures (such as the RMSD) and statistical goodness-of-fit tests, as we have done in this paper, can enrich the data analysis. The magnitude-of-discrepancy measure gives an interpretation in terms of previous data. As is well known, a statistic can (almost) always be made significant by collecting enough data. A magnitude-of-discrepancy measure can then indicate whether the effect found is worthwhile or is simply a matter of too much power. On the other hand, magnitude-of-discrepancy measures can only be compared mutually (not with respect to a statistical distribution). Our analysis has shown that, indeed, a magnitude of discrepancy in the order of .1 is significant, while a discrepancy in the order of .05 is not, as had been assumed before in FLMP articles. Whether the same holds for other models and different sample sizes can only be investigated by a systematic comparison of formal and discrepancysize measures.

As noted before, a goodness-of-fit statistic can also be evaluated in the FLMP model. One can, therefore, also compare magnitude-of-discrepancy measures with goodness-of-fit statistics in the FLMP model. This is, however, an uncommon practice in FLMP publications, so there is no research tradition in this field. Together with the flexibility arguments presented above, this indicates that the Rasch model has some advantages when used with the kinds of experiments to which the FLMP applies.

\section{SUMMARY}

We have shown that the Rasch model provides an easy and flexible way to analyze experimental data. In partic- ular, we fitted three models to see how the data might be summarized best. Such model construction is a straightforward procedure in Rasch-type models. For each model, goodness-of-fit statistics were calculated. Then it was shown that the formal and the discrepancy-size measures yield comparable results, and it was argued that comparing both types of measures is a useful way to arrive at conclusions about psychological data.

\section{REFERENCES}

ANDERSEN, E. B. (1980). Discrete statistical models with social science applications. Amsterdam: North-Holland.

Crowther, C. S., Batchelder, W. H., \& Hu, X. (1995). A measurementtheoretic analysis of the fuzzy logic model of perception. Psychological Review, 102, 396-408.

Fischer, G. H. (1973). The linear logistic test model as an instrument in educational research. Acta Psychologica, 37, 359-373.

Fischer, G. H. (1995). The linear logistic test model. In G. H. Fischer \& I. W. Molenaar (Eds.), Rasch models: Their foundations, recent developments and applications (pp. 131-155). New York: Springer-Verlag.

HABERMAN, S. J. (1977). Maximum likelihood estimates in exponential response models. Annals of Statistics, 5, 815-841.

HAMPTON, J. A. (1987). Inheritance of attributes in natural concept conjunctions. Memory \& Cognition, 15, 55-71.

HAMPTON, J. A. (1988). Overextension of conjunctive concepts: Evidence for a unitary model of concept typicality and class inclusion. Journal of Experimental Psychology: Learning, Memory, \& Cognition, 14, 12-32.

LUCE, R. D. (1959). Individual choice behavior. New York: Wiley.

MasSaro, D. W., \& HaRY, J. M. (1986). Addressing issues in letter recognition. Psychological Research, 48, 123-132.

OdEN, G. C. (1977). Fuzziness in semantic memory: Choosing exemplars of subjective categories. Memory \& Cognition, 5, 198-204.

ODEN, G. C. (1979). A fuzzy logical model of letter identification. Journal of Experimental Psychology: Human Perception \& Performance, 5, 336-352.

Ogasawara, H. (1996). Rasch's multiplicative Poisson model with covariates. Psychometrika, 61, 73-92.

RAsch, G. (1966). An item analysis which takes individual differences into account. British Journal of Mathematical \& Statistical Psychology, $19,49-57$.

Storms, G., De Boeck, P., Van Mechelen, I., \& Geeraerts, D. (1993). Dominance and noncommutativity effects in concept conjunctions: Extensional or intensional basis? Memory \& Cognition, 21, 752-762.

Storms, G., De Boeck, P., Van Mechelen, I., \& Ruts, W. (1996). The dominance effect in concept conjunctions: Generality and interaction effects. Journal of Experimental Psychology: Learning, Memory, \& Cognition, 22, 1-15.

Thissen, D., \& STEINBERG, L. (1986). A taxonomy of item response models. Psychometrika, 51, 567-577.

ZADEH, L. A. (1982). A note on prototype theory and fuzzy sets. Cognition, 12, 291-297.

\section{NOTES}

1. In order to avoid confusion, it should be noted at this point that, in the following, we do not refer to the fuzzy set approach to conceptual conjunctions as discussed by Zadeh (1982).

2. Available from the first author.
(Manuscript received October 28, 1996; revision accepted for publication July $8,1997$. 